\author{
Matthew C. Evans \\ Josephine Barnes \\ Casper Nielsen \\ Lois G. Kim \\ Shona L. Clegg \\ Melanie Blair \\ Kelvin K. Leung \\ Abdel Douiri \\ Richard G. Boyes \\ Sebastien Ourselin \\ Nick C. Fox \\ and the Alzheimer's Disease \\ Neuroimaging Initiative
}

Received: 14 April 2009

Revised: 6 July 2009

Accepted: 22 July 2009

Published online: 16 September 2009

(C) European Society of Radiology 2009

M. C. Evans · J. Barnes - C. Nielsen ·

L. G. Kim - S. L. Clegg - M. Blair ·

K. K. Leung · A. Douiri · R. G. Boyes

S. Ourselin - N. C. Fox $(\bowtie)$

Dementia Research Centre,

UCL Institute of Neurology,

Queen Square,

London, WC1N 3BG, UK

e-mail: nfox@dementia.ion.ucl.ac.uk

Tel.: +44-845-1555000

Fax: +44-20-76762066

L. G. Kim

London School of Hygiene

and Tropical Medicine,

London, UK

K. K. Leung $\cdot$ S. Ourselin

Centre for Medical Image Computing,

University College London,

London, UK

\title{
Volume changes in Alzheimer's disease and mild cognitive impairment: cognitive associations
}

\begin{abstract}
Objective: To assess the relationship between MRI-derived changes in whole-brain and ventricular volume with change in cognitive scores in Alzheimer's disease (AD), mild cognitive impairment (MCI) and control subjects. Material and
\end{abstract} methods: In total 131 control, 231 $\mathrm{MCI}$ and $99 \mathrm{AD}$ subjects from the Alzheimer's Disease Neuroimaging Initiative (ADNI) cohort with T1weighted volumetric MRIs from baseline and 12-month follow-up were used to derive volume changes. Mini mental state examination (MMSE), Alzheimer's disease assessment scale (ADAS)-cog and trails test changes were calculated over the same period. Results: Brain atrophy rates and ventricular enlargement differed between subject groups $(p<0.0005)$ and in MCI and AD were associated with MMSE changes. Both measures were additionally associated with ADAS-cog and trails-B in MCI patients, and ventricular expansion was associated with ADAS-cog in $\mathrm{AD}$ patients. Brain atrophy $(p<0.0005)$ and ventricular expansion rates $(p=0.001)$ were higher in MCI subjects who progressed to $\mathrm{AD}$ within 12 months of follow-up compared with MCI subjects who remained stable. MCI subjects who progressed to $\mathrm{AD}$ within 12 months had similar atrophy rates to $\mathrm{AD}$ subjects. Conclusion: Whole-brain atrophy rates and ventricular enlargement differed between patient groups and healthy controls, and tracked disease progression and psychological decline, demonstrating their relevance as biomarkers.

Keywords Alzheimer's disease . Mild cognitive impairment · MRI · Neuropsychology $\cdot$ Dementia

\section{Introduction}

Alzheimer's disease (AD) is the most common cause of dementia, estimated to affect 24 million people worldwide [1]. AD is a neurodegenerative disorder which typically has an insidious initial impairment in memory, extending to multiple domains as the disease progresses [2]. This is mirrored by pathology, with neurofibrillary tangles initially being limited to the hippocampus and entorhinal cortex, spreading to other limbic structures and eventually the whole cerebral cortex [3].

Amnestic mild cognitive impairment (MCI) describes subjects who show measurable memory deficits but do not meet $\mathrm{AD}$ criteria [4]. MCI therefore represents an opportunity to treat those who may progress to $\mathrm{AD}$ before widespread cognitive deficits are manifest.

A characteristic macroscopic consequence of $\mathrm{AD}$ pathology is brain atrophy, visible on MRI. At a group level $\mathrm{AD}$ is associated with smaller brain volumes and larger ventricles than healthy age-matched controls, while MCI groups have intermediate volumes [5]. However, there is overlap between these measures due to the natural variation in cerebral volumes, morphology and physiological state at the time of MRI. Rates of volume change may also discriminate between groups, reflecting differences in rates of neuronal degeneration. 
Although it is not possible to measure longitudinal neuronal loss directly in vivo, change in cerebral volume (atrophy rate) can be measured using MRI. This can either be calculated indirectly using estimates of regional or whole-brain volumes at two time points which requires two measurements with associated errors (e.g. 12-month interval), or by directly measuring volume change over time with methods such as the boundary shift integral (BSI) which requires one measure with associated error $[6,7]$. Direct methods have been shown to be more precise than indirect methods [8]. Measures of volume loss from MRI have been used to assess atrophy in $\mathrm{AD}[6,9]$, and to differentiate MCI and $\mathrm{AD}$ from controls [10-12]. Longitudinal studies show normal controls to have age-related cerebral atrophy rates of around $0.5 \%$ per year in subjects aged $65-75[13,14]$; while atrophy rates in $\mathrm{AD}$ are around $2 \%$ per year [15]. Rates of brain atrophy and ventricular enlargement have also been proposed as potential biomarkers of disease progression in trials. We wished to measure rates of change in brain and ventricular volumes and assess their relationship with cognitive measures and clinical progression from MCI to $\mathrm{AD}$.

The Alzheimer's Disease Neuroimaging Initiative (ADNI) is a multicentre study of $\mathrm{AD}$ and MCI patients and control subjects who received clinical, neuropsychological and imaging assessments at 6- or 12-month intervals (see http:// adni-info.org for full protocol). This study used baseline and 12-month assessments of ADNI subjects (a) to measure whole-brain and ventricular change in (i) $\mathrm{AD}$, (ii) $\mathrm{MCI}$ and (iii) healthy age-matched controls; (b) to assess the association of atrophy rates with cognitive change; and (c) to compare brain atrophy and ventricular expansion rates in MCI subjects who converted to AD within 12 months after baseline, with those who remained stable over this period.

\section{Materials and methods}

\section{Subject recruitment and assessment}

Each data acquisition site (http://adni-info.org/images/ stories/map_of_adni_sites_feb2006.pdf) first obtained institutional review board approval before collecting any data, and all subjects recruited gave informed written consent. Inclusion/exclusion criteria for healthy controls and $\mathrm{AD}$ subjects were similar to previous protocols (http://www.adni-info.org/images/stories/Documentation/ adni_protocol_03.02.2005_ss.pdf) [16]. All MCI subjects were amnestic and all AD subjects met standard criteria for a diagnosis of AD (NINCDS-ADRDA criteria for probable AD at baseline [17]). Each subject's diagnosis was reviewed at 6 months and 1 year. Subjects were included in this study if they had baseline and 12-month follow-up 1.5-T MR examination data available by June 2008 and both MR examinations passed standardised quality control. The MCI group were subdivided into two subgroups: converters referred to those subjects who progressed to meet criteria for AD within the 12-month follow-up period, whereas stable referred to those who maintained a diagnosis of amnestic MCI.

Image acquisition

MR images were acquired with a standardised protocol [18] on 1.5-T MRI units from Siemens Medical Solutions and General Electric Healthcare. MR protocols included high-resolution (typically $1.2 \times 1.25 \times 1.25 \mathrm{~mm}^{3}$ voxels) volumetric $\mathrm{T} 1$-weighted, inversion recovery prepared, structural images obtained sagittally (http://www.adni-info.org/ images/stories//mritrainingmanualv1.pdf for more details). Before the MR images were uploaded to the central image repository, images were gradwarp-corrected for (a) distortion due to gradient non-linearity [19], and (b) corrected for image intensity non-uniformity using N3 [18, 20] (all images), and B1 non-uniformity $[18,21]$ (where required, depending on the coils used) and (c) scaling-corrected based on phantom measures.

\section{Semi-automated segmentation of brains}

MR studies were downloaded from the website of the Laboratory of NeuroImaging (LONI, http://www.loni.ucla. edu/ADNI/) and transferred onto multiprocessor Intel Xeon-based workstations running 32-bit Linux. The image viewing and analysis software MIDAS [22] was used for whole-brain and ventricular semi-automated segmentation. Baseline whole-brain segmentation involved a semi-automated technique with manual checking and editing as necessary [22]. The intra-class correlation coefficient for inter-rater reliability (ICC) was greater than 0.99 calculated from 11 people segmenting five subjects' MR data. The ICC values for intra-rater reliability were all greater than 0.99 in 11 raters, segmenting five MR examinations twice. Baseline brain regions were propagated onto the follow-up MR data sets using affine and free-form deformation-based non-rigid registration [23, 24].

\section{Semi-automated segmentation of ventricles}

Baseline and follow-up MR examinations were registered to standard space (Montreal Neurological Institute 305 [25]) by estimating a nine-degrees of freedom (DoF) transformation over the brain region [24] but only applying the first six DoF (i.e. no scalings). An upper threshold of $60 \%$ of mean brain intensity was used to delineate the ventricle region, including the whole of the lateral ventricles and temporal horn, with manual editing performed where necessary. 
Boundary shift integral

For both brains and ventricles, the follow-up MR data and region were registered to the baseline using a nine-DoF transformation [24]. Brains were then differentially biascorrected [26] and then both whole-brain (BBSI) and ventricular (VBSI) BSIs were calculated [6]. BBSI was expressed as an atrophy rate: annualised percentage change in brain volume; VBSI was expressed as annualised ventricular expansion in millilitres as ventricles can not expand infinitely and normal variation in volumes is large, making assessment of these changes on an absolute scale more appropriate [14].

\section{Psychological data}

All subjects received a battery of psychological testing at baseline and 12 months. Only the mini mental state examination (MMSE) [27], the Alzheimer's disease assessment scale-cognitive subsection (ADAS-cog) [28] and trails-making (A and B) [29] measures were considered and assessed in this study. The MMSE is a commonly reported measure of global cognitive function and is marked out of 30, with a score of 24 or less being consistent with mild AD. ADAS-cog is a composite global measure of cognitive function marked out of 70 , with a high score being indicative of a greater deficit. By contrast, trails A and $\mathrm{B}$ are tests where subjects are required to join either consecutive numbers with a line (1-2-3-4 etc., part A) or consecutive numbers and letters $(1-\mathrm{A}-2-\mathrm{B}-3-\mathrm{C}$ etc., part B). Part A tests functions including visual search, speed of search, and processing of sequences, whereas part B includes the additional complexity of set-switching. Annual changes in neuropsychology scores were calculated as the change in score divided by the time between baseline and 12-month follow-up.

\section{Statistical analysis}

All statistical analysis was performed using STATA version 10. One-way ANCOVA was used to test whether percentage change in whole-brain volume (on a logarithmic scale) and ventricular expansion (on an arithmetic scale) differed by patient group (controls, MCI and AD), accounting for age, gender and baseline volume. No adjustment for premorbid brain size was made to brain volume owing to this being used as a covariate only. To account for unequal variances, robust standard errors were used. Post hoc pairwise tests were applied to compare patient groups.

One-way ANCOVA was also used to investigate group differences in annualised change scores from each of the neuropsychological tests (MMSE, ADAS-cog, trails A and B), similarly adjusted for baseline, age and gender and with robust standard errors. Ceiling effects in MMSE and trails A and B were also accounted for using binary indicators for those with baseline ceiling scores and those increasing to ceiling at follow-up. No individuals had ceiling scores in ADAS-cog at baseline or follow-up. No individuals reached floor scores in any cognitive measure at baseline or follow-up although two subjects who had a score of " 0 " in trails B were treated as having missing values.

Multivariable linear regression was used to investigate the relationship between change in the neuropsychological test scores (incorporating jump to ceiling) and brain atrophy rates and ventricular expansion. These analyses were performed separately by patient group adjusted for baseline volume, baseline neuropsychological score, age, gender and ceiling effects.

Differences in brain atrophy rates and ventricular enlargement between those MCI subjects who converted to $\mathrm{AD}$ within the period between MR examinations and those who did not were investigated with a regression model, adjusting for baseline volumes, age and gender and with robust standard errors.

\section{Results}

Subjects included

All MR studies received by June 2008 (120 AD, 275 MCI and 163 controls) were reviewed for quality; $21 \mathrm{AD}, 40$ $\mathrm{MCI}$ and 32 control subjects were excluded (controls= $19.6 \%, \mathrm{MCI}=14.6 \%, \mathrm{AD}=17.5 \% ; p=0.4)$ due to motion artefact or poor MR data set pair quality. This left $99 \mathrm{AD}$ subjects with a mean (standard deviation, SD) age of 75.3 (6.9) years; 235 MCI subjects aged 74.4 (7.1) years and 131 controls aged 76.0 (5.1) years; there was some evidence of a difference over all groups in terms of age $(p=0.08)$ and gender $(p=0.009)$, with a higher male proportion in the MCI group (see Table 1 for subject group demographics).

Table 2 and Fig. 1 show brain atrophy rates and ventricular expansion in the three groups. Both measures show control $<\mathrm{MCI}<\mathrm{AD}$ with considerably less betweensubject variation in the control group.

Annualised brain atrophy rates (\% change/year, $p<0.0005)$ and ventricular enlargement $(p<0.0005)$ differed between controls, MCI and AD subjects, after adjusting for baseline volumes, age and gender. In these models there was no evidence of an effect of gender on brain atrophy $(p=0.9)$ or ventricular expansion $(p=0.3)$; however, there was evidence for increasing age to be associated with lower whole-brain atrophy $(p=0.04)$ and ventricular expansion $(p<0.0005)$ rates. There was some evidence of a qualitative interaction between age and group $(p<0.05$, joint interaction tests) with group differences decreasing with age, but these effects were not large. 
Table 1 Baseline information by patient group

\begin{tabular}{|c|c|c|c|c|c|}
\hline & \multirow[b]{2}{*}{ Controls } & \multicolumn{3}{|l|}{ MCI } & \multirow[b]{2}{*}{$\mathrm{AD}$} \\
\hline & & Non-converters & Converters & All MCI & \\
\hline$N$ & 131 & 188 & 43 & 231 & 99 \\
\hline Age, years (mean (SD)) & $76.0(5.1)$ & $74.4(7.2)$ & $74.5(6.4)$ & $74.4(7.1)$ & $75.3(6.9)$ \\
\hline$\%$ male & 47 & 63 & 60 & 63 & 51 \\
\hline MMSE (mean (SD)) & $29.2(1.0)$ & $27.0(1.8)$ & $26.6(1.8)$ & $27.0(1.8)$ & $23.5(1.8)$ \\
\hline ADAS-cog (mean (SD)) & $5.9(3.0)$ & $11.1(4.1)$ & $13.6(4.9)$ & $11.6(4.4)$ & $18.0(6.0)^{\mathrm{b}}$ \\
\hline Trails-A (median (IQR)) & $33(28,42)$ & $37(30,47)$ & $45(35,56)$ & $38(31,49)$ & $53(41,81)$ \\
\hline Trails-B (median (IQR)) & $77(60,97)$ & $102(73,153)^{\mathrm{a}}$ & $133(87,212)$ & $105(75,162)$ & $186(112,300)^{\mathrm{c}}$ \\
\hline Brain volume, ml (mean (SD)) & $1,049(103)$ & $1,059(117)$ & $1,002(85)$ & $1,048(114)$ & $1,007(111)$ \\
\hline Ventricular volume, ml (mean (SD)) & $38(22)$ & $45(23)$ & $52(25)$ & $46(24)$ & $52(27)$ \\
\hline
\end{tabular}

$I Q R$ interquartile range

${ }^{a}$ Missing for four individuals

${ }^{\mathrm{b}}$ Missing for one individual

${ }^{\mathrm{c}}$ Missing for 17 individuals

Psychological data

Table 2 shows baseline and 0-12 month change for the MMSE, ADAS-cog and trails A and B scores in the three groups. There was strong evidence for a difference in change scores adjusted for baseline score, age and gender (and baseline ceiling scores for MMSE and trails measures) in all cognitive measures for controls compared with MCI subjects (MMSE $p<0.0005$, ADAS- $\operatorname{cog} p<0.0005$, trails-A $p<0.0005$, trails-B $p<0.0005)$ and for controls compared with AD patients (MMSE $p<0.0005$, ADAS$\operatorname{cog} p<0.0005$, trails-A $p<0.0005$, trails-B $p<0.0005$ ). There was also evidence for a difference between MCI and AD patients in MMSE change scores $(p=0.01)$, ADAS-cog $(p<0.0005)$ and trails-B $(p<0.0005)$, but not trails-A $(p=0.1)$.
Associations between atrophy rates and cognitive measures

There was strong evidence for an association between brain atrophy rates and change in MMSE in both MCI $(p<0.0005)$ and $\mathrm{AD}(p=0.002)$ patients. There was also strong evidence for an association between brain atrophy and ADAS-cog change in MCI $(p=0.0001)$ and borderline evidence in $\mathrm{AD}(p=0.06)$ patients. There was no evidence of an association between brain atrophy rates and trails A change in MCI $(p=0.2)$ or AD $(p=0.2)$, and whilst there was an association between brain atrophy rates and trails B in MCI $(p<0.0005)$, there was no evidence of association in $\operatorname{AD}(p=0.4)$.

There was strong evidence for an association between ventricular expansion and MMSE change in MCI

Table 2 Raw change scores by patient group

\begin{tabular}{|c|c|c|c|c|c|}
\hline & \multirow[b]{2}{*}{ Controls } & \multicolumn{3}{|l|}{ MCI } & \multirow[b]{2}{*}{$\mathrm{AD}$} \\
\hline & & Non-converters & Converters & All MCI & \\
\hline$N$ & 131 & 188 & 43 & 231 & 99 \\
\hline Time to follow-up, years (mean (SD)) & $1.07(0.06)$ & $1.07(0.06)$ & $1.04(0.05)$ & $1.07(0.06)$ & $1.07(0.06)$ \\
\hline MMSE change $(\text { mean }(\mathrm{SD}))^{\mathrm{a}}$ & $-0.02(1.23)$ & $-0.02(1.23)$ & $-2.06(3.62)$ & $-0.85(2.45)$ & $-2.06(3.62)$ \\
\hline ADAS-cog change (mean $(\mathrm{SD}))^{\mathrm{a}}$ & $-0.53(2.70)$ & $0.75(4.06)$ & $2.93(4.94)$ & $1.16(4.31)$ & $3.53(5.42)^{\mathrm{c}}$ \\
\hline Trails-A change (mean (SD) $)^{\mathrm{a}}$ & $-1.9(9.4)$ & $2.3(14.6)$ & $1.2(13.1)$ & $2.1(14.3)$ & $4.0(19.1)$ \\
\hline Trails-B change (mean $(\mathrm{SD}))^{\mathrm{a}}$ & $-5.7(33.4)$ & $8.3(48.8)^{\mathrm{b}}$ & $33.1(68.7)$ & $13.0(53.8)$ & $32.0(58.1)^{\mathrm{d}}$ \\
\hline Brain atrophy, $\%$ loss per year (mean $(\mathrm{SD}))^{\mathrm{a}}$ & $0.49(0.59)$ & $0.92(0.83)$ & $1.61(1.08)$ & $1.05(0.92)$ & $1.50(0.92)$ \\
\hline VBSI, ml expansion per year (mean (SD) $)^{\mathrm{a}}$ & $1.30(1.41)$ & $2.77(2.43)$ & $4.51(3.43)$ & $3.09(2.72)$ & $4.40(2.99)$ \\
\hline
\end{tabular}

${ }^{\mathrm{a} C h a n g e}$ per year of follow-up

${ }^{\mathrm{b}}$ Missing for four individuals

${ }^{\mathrm{c}}$ Missing for one individual

${ }^{\mathrm{d}}$ Missing for 17 individuals, including 2 with follow-up scores of 0 and 2 with follow-up scores of -1 (missing indicator) 
Fig. 1 Whole-brain atrophy rates (left) and ventricular expansion rates (right), by patient group (unadjusted for baseline volumes)

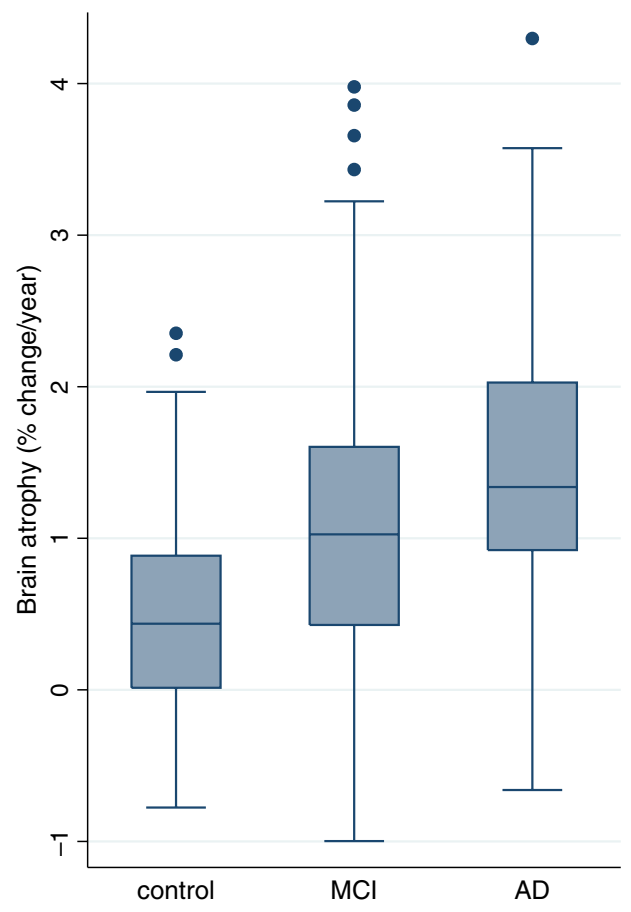

$(p<0.0005)$ and $\operatorname{AD}(p=0.002)$. There was also strong evidence for an association between ventricular expansion and ADAS-cog change in MCI $(p<0.0005)$ and AD $(p=0.008)$. There was no evidence of association between ventricular expansion and trails A change in either MCI $(p=0.2)$ or $\mathrm{AD}(p=0.3)$; however, there was evidence for an association between ventricular expansion and trails B change in MCI $(p=0.003)$, although no evidence in $\mathrm{AD}$ $(p=0.2)$.

\section{MCI converters and non-converters}

MCI converters had rates of brain atrophy ( $\%$ change/year, $p<0.0005)$ and ventricular expansion $(p=0.001)$ that were both approximately $50 \%$ higher than non-converters (see Table 2 and Fig. 2), after adjustment for baseline volumes, age and gender. In these models there was no evidence of an effect of gender on brain atrophy $(p=0.4)$ or ventricular expansion rates $(p=0.6)$; however, there was borderline evidence for increasing age to be associated with lower whole-brain rates $(p=0.06)$ and strong evidence for increasing age to be associated with lower ventricular expansion $(p<0.0005)$ rates. There was no evidence that group differences varied by age $(p>0.05$, interaction tests).

MCI non-converters also differed from both controls (brain atrophy rates $(p<0.0005)$ and ventricular expansion $(p<0.0005))$ and $\mathrm{AD}$ (brain atrophy rates $(p<0.0005)$ and ventricular expansion $(p<0.0005))$. MCI converters differed from controls (brain atrophy rates $(p<0.0005)$ and ventricular expansion $(p<0.0005))$ but not $A D$ patients (neither brain atrophy rates $(p=0.6)$ or ventricular expansion $(p=0.9))$.

\section{Discussion}

In this large multicentre study we found mean (SD) wholebrain loss at $1.5 \%$ year $(0.9 \%)$ in $\mathrm{AD}$ to be approximately three times higher than the control rates of $0.5 \% /$ year $(0.6 \% / y e a r)$ in controls whilst the MCI group as a whole had an intermediate rate of approximately $1.1 \% /$ year $(0.9 \% /$ year $)$. Some studies have reported higher atrophy rates for $\mathrm{AD}$ subjects although subjects were often more severely affected at the time of imaging. For example, O'Brien et al. reported a brain atrophy rate of $2.0 \% /$ year $(0.9 \% /$ year) in patients with a mean age of 75 and mean MMSE of 16 [9]; Fox et al. reported a brain atrophy rate of $2.4 \% /$ year $(1.1 \% /$ year $)$ for a mean age of 65 and mean MMSE of 20 [30]; Archer et al. reported a brain atrophy rate of $1.8 \% /$ year $(1.4 \% /$ year) for a mean age of 66 and mean MMSE 21 [31]; Wang et al. reported a brain atrophy rate of $2.4 \%$ year (1.2\%/year) for a mean age of 67 and mean MMSE 20 [32]; Sluimer et al. reported a rate of $1.9 \% /$ year $(0.9 \% /$ year $)$ for a mean age of 67 years and mean MMSE 22 [12]. The mean MMSE in ADNI is higher than the studies reported above, as ADNI enrolled at an early disease stage. Atrophy rate in early disease has been reported to be lower and this rate increases with severity [5, 33-35]. Another study has reported a lower rate of atrophy than our study; Jack et al. reported slow-progressing ADs 
Fig. 2 Whole-brain atrophy rates (left) and ventricular expansion rates (right), in MCI patients who subsequently progressed to $\mathrm{AD}$ within 1 year of follow up (converters) and those that did not (nonconverters) (unadjusted for baseline volumes)
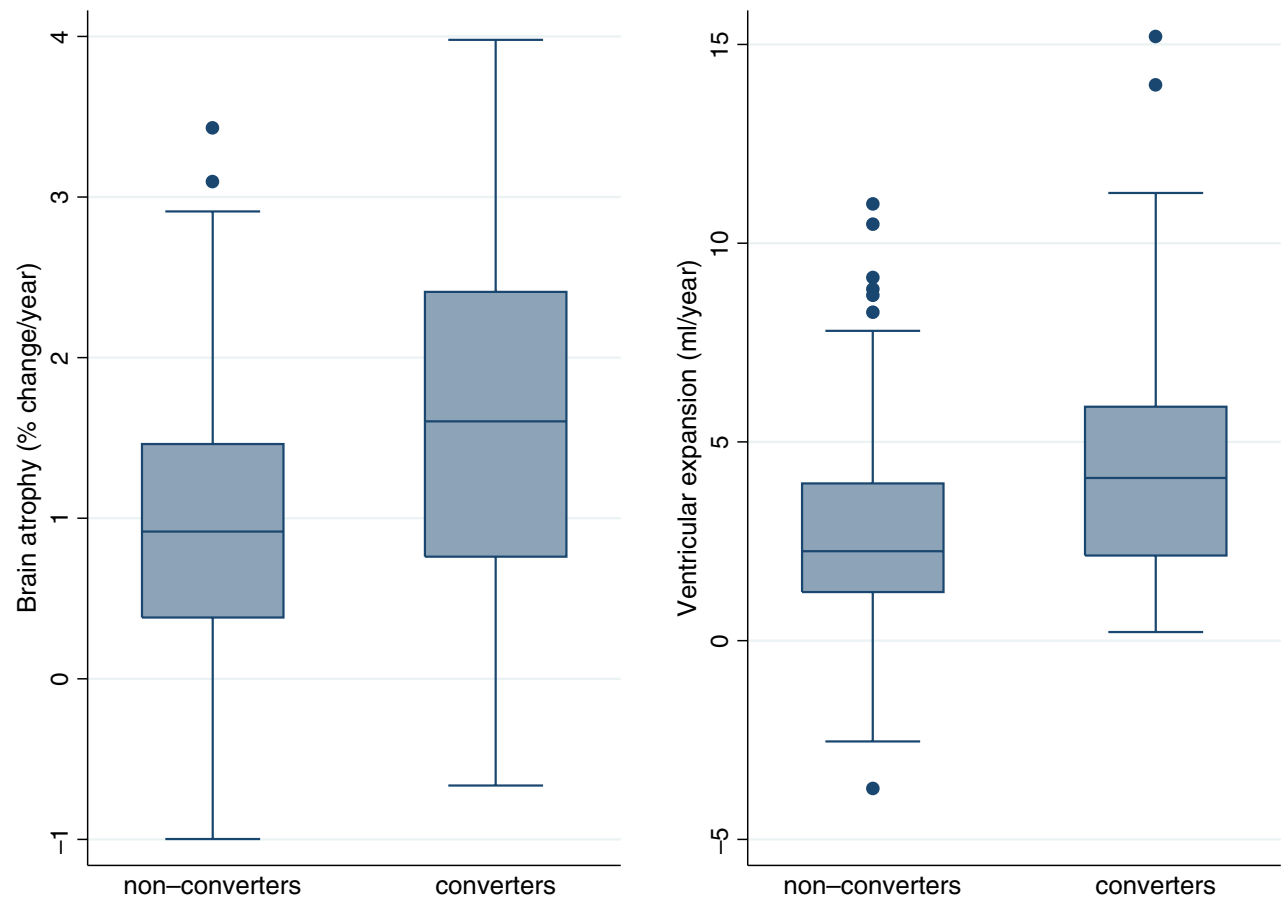

having a median (interquartile range) atrophy rate of $0.6 \% /$ year $(0.7 \%$ year) and fast-progressing patients having an atrophy rate of $1.4 \% /$ year (1.1\%/year) [10].

Interestingly the MCI subjects who progressed to $\mathrm{AD}$ during the follow-up had a brain atrophy rate of $1.6 \%$ year $(1.1 \% /$ year $)$ which was very similar to the $\mathrm{AD}$ group and about twice that of the MCI subjects who did not progress to $\mathrm{AD} 0.9 \% /$ year $(0.8 \% /$ year). The MCI subjects who did not progress had a higher brain atrophy rate than controls, implying that some of these MCI subjects were heading towards an AD atrophy rate. Jack et al. reported differences in atrophy rates (median (interquartile range)) for stable vs. converting MCI subjects as $0.4 \% /$ year $(0.4 \% /$ year $)$ and $0.8 \% /$ year $(0.5 \% /$ year $)$, respectively [10]. Another study reported an overall mean (SD) atrophy rate in $\mathrm{MCI}$ of $0.7 \% /$ year $(0.7 \% /$ year) [11]. One recent paper has also demonstrated that because MCI atrophy rates accelerate in those subjects who convert to $\mathrm{AD}$ the calculated rate in converters in any study may therefore be dependent upon proximity to conversion [5]. A further paper has shown that higher whole-brain atrophy rates are associated with an increased risk of converting to $\mathrm{AD}$ from MCI [12].

In this study, rates of ventricular enlargement had a similar relationship to whole brain: mean (SD) rate was $4.4 \mathrm{ml} /$ year (3.0 ml/year) in AD patients which was just over three times the rate of controls which was $1.3 \mathrm{ml} /$ year ( $1.4 \mathrm{ml} /$ year). Rates in the MCI group were between control and $\mathrm{AD}$ rates at $3.1 \mathrm{ml} /$ year $(2.7 \mathrm{ml} /$ year $)$. Many studies report relative (as a percentage of baseline volume) rather than absolute atrophy rates for ventricles; our rates using this method were $10.4 \% /$ year (6.4\%/year) in $\mathrm{AD}$ patients, $8.2 \% /$ year $(8.0 \% /$ year) in MCI subjects and 3.9\%/year (4.8\%/year) in controls. Reported rates of ventricular enlargement in $\mathrm{AD}$ are varied, ranging from 4 to $10 \mathrm{ml} / \mathrm{year}$. Silbert et al. reported a ventricular change of $5.5 \mathrm{ml} /$ year (3.2 ml/year) for a mean age of 82 and final MMSE of 12 [36]; Schott et al. reported a ventricular change of $4.3 \mathrm{ml} /$ year (2.4 ml/year) for a mean age 70 and MMSE of 20 [37]; Kaye et al. reported a ventricular change of $9.9 \mathrm{ml} /$ year $(5.8 \mathrm{ml} /$ year) in mild $\mathrm{AD}$ for a mean age of 76 and mean MMSE 22 [38]; Wang et al. reported a ventricular change of $8.2 \mathrm{ml} /$ year (no SD given) for a mean age of 67 and mean MMSE of 19.6 [32]. Silbert et al. included older subjects than our study and with a much lower MMSE, yet reported ventricular rates that are comparable [36]. Differences in reported rates may relate to methodology, most importantly how much of the ventricular system was included. In terms of MCI, various rates of ventricular change have been noted, some much lower than reported here; for example, Jack et al. reported a ventricular change of 3.3\%/year (2.3\%/year) for a mean follow-up age of 80 and mean follow-up MMSE of 26 [11]. This is consistent with rates that are lower than some of our previous studies; this may be due to patients being more mildly affected, but it is possible that a number of subjects do not have AD.

We showed that in both $\mathrm{MCI}$ and $\mathrm{AD}$ groups, brain and ventricular changes were associated with change over 12 months in both the MMSE and ADAS-cog measures although this was borderline in brain rates with ADAS-cog in $\mathrm{AD}$, and these results are consistent with previous 
reports $[10,12,15,39-41]$. The associations may be explained by the nature of the neuropsychological tests: both MMSE and ADAS-cog are tests of global functioning, spanning many domains localised to multiple cerebral regions in different lobes and sub-cortical structures, giving a strong association with global atrophy measures. We did not show any association with trails A or B scores for the AD group and only for MCI with trails B. The trail-making test is a more focal rather than global cognitive measure, testing aspects of visual search, executive function and attention, which are localised mainly to frontal (especially pre-frontal) and parietal lobes. Furthermore, there was huge variability in change in trails scores in all patient groups.

We also showed that both brain atrophy rates and ventricular expansion were higher in MCI subjects that converted within the follow-up period compared with those that remained stable. MCI converters were very similar to the clinically diagnosed $\mathrm{AD}$ group in terms of brain atrophy rates and ventricular expansion, which along with similar cognitive profiles (Table 1) shows AD-like presentation before/during conversion. Together these results suggest a gradual adoption of AD-type cerebral and cognitive profile with advancing disease progression. Non-converters were different from both AD and controls, placing them between these two groups, presumably reflecting that some subjects are further from conversion or may not convert in the future.

The data presented here are from a large multicentre cohort typical of those recruited into therapeutic trials. We conclude that brain and ventricular atrophy rates were higher in MCI than controls and higher in AD than MCI although there remained overlap between groups, with a similar pattern shown for baseline and change scores for neuropsychology. A strong brain-behaviour relationship was demonstrated by the associations of the neuropsycho- logical variables with brain and ventricular change. Those MCI subjects who converted to AD within 12 months of baseline assessment had atrophy rates similar to AD subjects. These findings suggest that MCI may be a useful group for use in clinical trials to test the efficacy of putative pharmacological agents, particularly if those subjects likely to progress could be separated from those likely to remain stable.

Acknowledgements This work was undertaken in University College London Hospitals/University College London, which received a proportion of funding from the UK Department of Health's National Institute of Health Research Biomedical Research Centres funding scheme. The Dementia Research Centre is an Alzheimer's Research Trust Co-ordinating Centre. Data were obtained from the Alzheimer's Disease Neuroimaging Initiative database (www.loni.ucla.edu/ADNI). A list of authors that contributed to varied aspects of design and implementation of the ADNI study, but did not contribute to the writing of this article can be found at http://www.loni.ucla.edu/ADNI/ About/About_InvestigatorsTable.shtml. ADNI is funded by the National Institute of Ageing, the National Institute of Biomedical Imaging and Bioengineering (NBIB), and the Foundation of the National Institutes of Health, contributions from the following companies and organisations: Pfitzer Inc., Wyeth Research, BristolMyers Squibb, Eli Lilly and Company, GlaxoSmithKline, Merck \& Co. Inc., AstraZeneca AB, Novartis Pharmaceuticals Corporation, the Alzheimer's Association, Eisai Global Clinical Development, Elan Corporation plc, Forest Laboratories, and the Institute for the Study of Ageing (ISOA), with participation from the U.S. Food and Drug Administration. N Fox is supported by the Medical Research Council (UK) Senior Clinical Fellowship. J Barnes is supported by Alzheimer's Research Trust (UK) Research Fellowship with the kind support of the Kirby Laing Foundation. K Leung was funded by a Technology Strategy Board Grant. The authors would like to thank Chris Frost for his statistical advice. Author contributions were as follows: Drafting and editing: MCE, JB, NCF, LGK; quality control of MRI images was carried out by SLC, MCE and MB; data download performed and MR data issues dealt with by $\mathrm{CN}, \mathrm{RB}$ and $\mathrm{KL} ; \mathrm{AD}$ and $\mathrm{RB}$ were responsible for developing the propagation technique used; MCE conducted the statistical analysis under supervision of LGK.

\section{References}

1. Ferri CP, Prince M, Brayne C, Brodaty $\mathrm{H}$, Fratiglioni L, Ganguli M, Hall K, Hasegawa K, Hendrie H, Huang Y, Jorm A, Mathers C, Menezes PR, Rimmer E, Scazufca M (2005) Global prevalence of dementia: a Delphi consensus study. Lancet 366:2112-2117

2. American Psychiatric Association (1994) Diagnostic and statistical manual of mental disorders, 4th edn (DSM-IV). American Psychological Association, Washington
3. Braak H, Braak E (1991) Neuropathological stageing of Alzheimer-related changes. Acta Neuropathol 82:239259

4. Petersen RC, Smith GE, Waring SC, Ivnik RJ, Tangalos EG, Kokmen E (1999) Mild cognitive impairment: clinical characterization and outcome. Arch Neurol 56:303-308

5. Jack CR Jr, Weigand SD, Shiung MM, Przybelski SA, O'Brien PC, Gunter JL, Knopman DS, Boeve BF, Smith GE, Petersen RC (2008) Atrophy rates accelerate in amnestic mild cognitive impairment. Neurology 70:1740 1752
6. Freeborough PA, Fox NC (1997) The boundary shift integral: an accurate and robust measure of cerebral volume changes from registered repeat MRI. IEEE Trans Med Imag 16:623-629

7. Fox NC, Freeborough PA, Rossor MN (1996) Visualisation and quantification of rates of atrophy in Alzheimer's disease. Lancet 348:94-97

8. Frost C, Kenward MG, Fox NC (2004) The analysis of repeated 'direct' measures of change illustrated with an application in longitudinal imaging. Stat Med 23:3275-3286 
9. O'Brien JT, Paling S, Barber R, Williams ED, Ballard C, McKeith IG, Gholkar A, Crum WR, Rossor MN, Fox NC (2001) Progressive brain atrophy on serial MRI in dementia with Lewy bodies, AD, and vascular dementia. Neurology 56:1386-1388

10. Jack CR Jr, Shiung MM, Gunter JL, O'Brien PC, Weigand SD, Knopman DS, Boeve BF, Ivnik RJ, Smith GE, Cha RH, Tangalos EG, Petersen RC (2004) Comparison of different MRI brain atrophy rate measures with clinical disease progression in AD. Neurology 62:591-600

11. Jack CR Jr, Shiung MM, Weigand SD, O'Brien PC, Gunter JL, Boeve BF, Knopman DS, Smith GE, Ivnik RJ, Tangalos EG, Petersen RC (2005) Brain atrophy rates predict subsequent clinical conversion in normal elderly and amnestic MCI. Neurology 65:1227-1231

12. Sluimer JD, van der Flier WM, Karas GB, Fox NC, Scheltens P, Barkhof F, Vrenken H (2008) Whole-brain atrophy rate and cognitive decline: longitudinal MR study of memory clinic patients. Radiology 248:590-598

13. Fox NC, Schott JM (2004) Imaging cerebral atrophy: normal ageing to Alzheimer's disease. Lancet 363:392394

14. Scahill RI, Frost C, Jenkins R, Whitwell JL, Rossor MN, Fox NC (2003) A longitudinal study of brain volume changes in normal aging using serial registered magnetic resonance imaging. Arch Neurol 60:989-994

15. Fox NC, Scahill RI, Crum WR, Rossor MN (1999) Correlation between rates of brain atrophy and cognitive decline in AD. Neurology 52:1687-1689

16. Aisen PS, Schafer KA, Grundman M, Pfeiffer E, Sano M, Davis KL, Farlow MR, Jin S, Thomas RG, Thal LJ (2003) Effects of rofecoxib or naproxen vs placebo on Alzheimer disease progression: a randomized controlled trial. JAMA 289:2819-2826

17. McKhann G, Drachman D, Folstein M, Katzman R, Price D, Stadlan EM (1984) Clinical diagnosis of Alzheimer's disease: report of the NINCDS-ADRDA work group under the auspices of Department of Health and Human Services Task Force on Alzheimer's Disease. Neurology 34:939-944
18. Jack CR Jr, Bernstein MA, Fox NC, Thompson P, Alexander G, Harvey D, Borowski B, Britson PJ, Whitwell L, Ward C, Dale AM, Felmlee JP, Gunter JL, Hill DL, Killiany R, Schuff N, FoxBosetti S, Lin C, Studholme C, DeCarli CS, Krueger G, Ward HA, Metzger GJ, Scott KT, Mallozzi R, Blezek D, Levy J, Debbins JP, Fleisher AS, Albert M, Green R, Bartzokis G, Glover G, Mugler J, Weiner MW (2008) The Alzheimer's Disease Neuroimaging Initiative (ADNI): MRI methods. J Magn Reson Imaging 27:685-691

19. Jovicich J, Czanner S, Greve D, Haley E, van der KA, Gollub R, Kennedy D, Schmitt F, Brown G, Macfall J, Fischl B, Dale A (2006) Reliability in multisite structural MRI studies: effects of gradient non-linearity correction on phantom and human data. Neuroimage 30:436-443

20. Sled JG, Zijdenbos AP, Evans AC (1998) A nonparametric method for automatic correction of intensity nonuniformity in MRI data. IEEE Trans Med Imag 17:87-97

21. Narayana PA, Brey WW, Kulkarni MV, Sievenpiper CL (1988) Compensation for surface coil sensitivity variation in magnetic resonance imaging. Magn Reson Imaging 6:271-274

22. Freeborough PA, Fox NC, Kitney RI (1997) Interactive algorithms for the segmentation and quantitation of 3-D MRI brain scans. Comput Methods Programs Biomed 53:15-25

23. Rueckert D, Sonoda LI, Hayes C, Hill DLG, Leach MO, Hawkes DJ (1999) Nonrigid registration using free-form deformations: application to breast MR images. IEEE Trans Med Imag 18:712721

24. Woods RP, Grafton ST, Holmes CJ, Cherry SR, Mazziotta JC (1998) Automated image registration: I. General methods and intrasubject, intramodality validation. J Comput Assist Tomogr 22:139-152

25. Mazziotta JC, Toga AW, Evans A, Fox P, Lancaster J (1995) A probabilistic atlas of the human brain: theory and rationale for its development. Neuroimage 2:89-101

26. Lewis EB, Fox NC (2004) Correction of differential intensity inhomogeneity in longitudinal MR images. Neuroimage 23:75-83

27. Folstein MF, Folstein SE, Mchugh PR (1975) Mini-mental state-practical method for grading cognitive state of patients for clinician. J Psychiatr Res 12:189-198
28. Rosen WG, Mohs RC, Davis K (1984) A new rating scale for Alzheimer's disease. Am J Psychiatry 141:13561364

29. Reitan RM, Wolfson D (1985) The Halstead-Reitan neuropsychological test battery: therapy and clinical interpretation. AZ Neuropsychological, Tucson

30. Fox NC, Cousens S, Scahill R, Harvey RJ, Rossor MN (2000) Using serial registered brain magnetic resonance imaging to measure disease progression in Alzheimer disease: power calculations and estimates of sample size to detect treatment effects [see comments]. Arch Neurol 57:339-344

31. Archer HA, Edison P, Brooks DJ, Barnes J, Frost C, Yeatman T, Fox NC, Rossor MN (2006) Amyloid load and cerebral atrophy in Alzheimer's disease: an 11C-PIB positron emission tomography study. Ann Neurol 60:145-147

32. Wang D, Chalk JB, Rose SE, de Zubicaray G, Cowin G, Galloway GJ, Barnes D, Spooner D, Doddrell DM, Semple J (2002) MR image-based measurement of rates of change in volumes of brain structures. Part II: application to a study of Alzheimer's disease and normal aging. Magn Reson Imaging 20:41-48

33. Scahill RI, Schott JM, Stevens JM, Rossor MN, Fox NC (2002) Mapping the evolution of regional atrophy in Alzheimer's disease: unbiased analysis of fluid-registered serial MRI. Proc Natl Acad Sci USA 99:4703-4707

34. Chan D, Janssen JC, Whitwell JL, Watt HC, Jenkins R, Frost C, Rossor MN, Fox NC (2003) Change in rates of cerebral atrophy over time in earlyonset Alzheimer's disease: longitudinal MRI study. Lancet 362:1121-1122

35. Mungas D, Harvey D, Reed BR, Jagust WJ, DeCarli C, Beckett L, Mack WJ, Kramer JH, Weiner MW, Schuff N, Chui HC (2005) Longitudinal volumetric MRI change and rate of cognitive decline. Neurology 65:565-571

36. Silbert LC, Quinn JF, Moore MM, Corbridge E, Ball MJ, Murdoch G, Sexton G, Kaye JA (2003) Changes in premorbid brain volume predict Alzheimer's disease pathology. Neurology 61:487-492 
37. Schott JM, Price SL, Frost C, Whitwell JL, Rossor MN, Fox NC (2005) Measuring atrophy in Alzheimer disease: a serial MRI study over 6 and 12 months. Neurology 65:119124
38. Kaye JA, Moore MM, Dame A, Quinn J, Camicioli R, Howieson D, Corbridge E, Care B, Nesbit G, Sexton G (2005) Asynchronous regional brain volume losses in presymptomatic to moderate AD. J Alzheimers Dis 8:51-56

39. Ridha BH, Anderson VM, Barnes J, Boyes RG, Price SL, Rossor MN, Whitwell JL, Jenkins L, Black RS, Grundman M, Fox NC (2008) Volumetric MRI and cognitive measures in Alzheimer disease: comparison of markers of progression. J Neurol 255:567-574
40. Nestor SM, Rupsingh R, Borrie M, Smith M, Accomazzi V, Wells JL, Fogarty J, Bartha R (2008) Ventricular enlargement as a possible measure of Alzheimer's disease progression validated using the Alzheimer's disease neuroimaging initiative database. Brain 131:2443-2454

41. Lehericy S, Marjanska M, Mesrob L, Sarazin M, Kinkingnehun S (2007) Magnetic resonance imaging of Alzheimer's disease. Eur Radiol 17:347362 